\title{
Cortical thickness across the cingulate gyrus in schizophrenia and its association to illness duration and memory performance
}

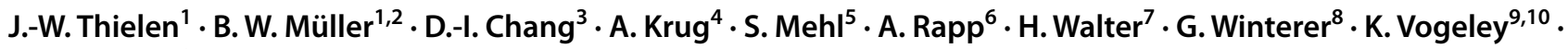 \\ S. Klingberg ${ }^{6} \cdot M$. Wagner $^{4} \cdot$ T. Kircher $^{4}$
}

Received: 16 April 2021 / Accepted: 29 November 2021 / Published online: 8 January 2022

(c) The Author(s) 2022

\begin{abstract}
Schizophrenia has been associated with structural brain abnormalities and cognitive deficits that partly change during the course of illness. In the present study, cortical thickness in five subregions of the cingulate gyrus was assessed in 44 patients with schizophrenia-spectrum disorder and 47 control persons and related to illness duration and memory capacities. In the patients group, cortical thickness was increased in the posterior part of the cingulate gyrus and related to illness duration whereas cortical thickness was decreased in anterior parts unrelated to illness duration. In contrast, cortical thickness was related to episodic and working memory performance only in the anterior but not posterior parts of the cingulate gyrus. Our finding of a posterior cingulate increase may point to either increased parietal communication that is accompanied by augmented neural plasticity or to effects of altered neurodegenerative processes in schizophrenia.
\end{abstract}

Keywords Schizophrenia $\cdot$ Cingulate gyrus $\cdot$ Magnetic resonance imaging $\cdot$ Neuropsychological assessment

\section{Introduction}

Cognitive deficits are considered to be a primary characteristic of schizophrenia [69]. Retrospective studies showed cognitive deficits to be among one of the first prodromal signs in individuals who were later diagnosed with schizophrenia $[32,74]$. Moreover, the importance of understanding cognitive dysfunction in schizophrenia is accentuated by the relative lack of cognition-specific treatment options [12]. Evidence suggests that there are discrete domains of

J.-W. Thielen

jan-willem.thielen@uni-due.de

1 Department of Psychiatry and Psychotherapy, LVR-Hospital Essen, Faculty of Medicine, University of Duisburg-Essen, Duisburg, Germany

2 Department of Psychology, University of Wuppertal, Wuppertal, Germany

3 Department of Psychiatry, LWL University Hospital Bochum, Ruhr University Bochum, Bochum, Germany

4 Department of Psychiatry and Psychotherapy, University of Bonn, Bonn, Germany

5 Department of Psychiatry and Psychotherapy and Center for Mind, Brain and Behavior (MCMBB), Philipps-University, Marburg, Germany cognitive impairment. An early study by Bilder et al. [8] reported mild to moderate deficits in verbal fluency, attention, processing speed and working memory but more severe deficits in executive functioning and episodic memory.

Studies assessing memory indicate that impairments in schizophrenia are common and disproportionately pronounced in comparison to the overall level of intellectual impairment [1, 23, 28, 61]. McKenna et al. [50] even proposed the existence of a "schizophrenic amnesia". However, it has been suggested that some aspects of memory may be

6 Department of Psychiatry and Psychotherapy, University of Tübingen, Tübingen, Germany

7 Division of Mind and Brain Research, Department of Psychiatry and Psychotherapy CCM, Charité-Universitätsmedizin Berlin, Corporate Member of Freie Universität Berlin, Humboldt-Universität Zu Berlin, and Berlin Institute of Health, Berlin, Germany

8 Department of Psychiatry and Psychotherapy, University of Berlin, Berlin, Germany

9 Department of Psychiatry and Psychotherapy, University of Cologne, Cologne, Germany

10 Institute of Neuroscience and Medicine, Cognitive Neuroscience (INM-3), Research Center Jülich, Jülich, Germany 
affected to a greater extent than others [1, 30]. For instance, studies in which tasks were matched for difficulty level, report greater impairment in recall as compared to recognition [1], verbal memory seemed to be more substantially impaired as compared to visual memory [73]. Whereas the different aspects of memory deficits in schizophrenia have been extensively studied, less is known about the course of memory dysfunction over time in schizophrenia. Initial evidence suggested that duration of illness, after removing variance related to e.g. age, is associated with several domains of memory $[65,69]$. For instance, longer duration of the disorder was associated with worse performance on verbal $[26,69]$ and visual $[26,65]$, Cuesta et al. [17] episodic memory tasks as well as working memory tasks [22, 26]. To possibly localize these deficits on a neurobiological level, brain morphology has been studied extensively. In this regard, Hulshoff Pol and Kahn [35] reviewed longitudinal studies of brain anatomy in schizophrenia and reported progressive reductions in brain tissue over the first 20 years of the disorder, with schizophrenia subjects showing more than twice the rate of tissue loss as compared to controls.

Post-mortem studies in patients with schizophrenia provided evidence for neuroanatomical abnormalities, in particular the cingulate gyrus [7, 13, 72]. Moreover, in vivo neuroimaging studies comparing patients with schizophrenia to healthy controls have shown evidence of decreased gray matter volume in the posterior cingulate gyrus [36, 68], the anterior cingulate gyrus [31, 38, 66, 67], and across the entire cingulate gyrus [51, 53, 80]. Beckmann and colleagues [6] performed a connectivity based parcellation of the cingulate cortex and related the different sub regions to specific functions based on peak activations of 171 functional magnetic resonance studies. With respect to memory functions, they found two spatially distinct regions of increased neural activation, namely in the dorsal anterior and in the posterior part of the cingulate gyrus. Moreover, the authors showed that the anterior activation cluster overlay with activation seen in studies of error, reward and conflict monitoring while the posterior activation cluster overlay with regions associated with processing of spatial information and visual imagination. Given these findings, one may hypothesize that different aspects of memory decline in patients with schizophrenia are associated with gray matter abnormalities across different sub-regions of the cingulate gyrus that worsen with duration of the illness.

In the present study, changes in cortical thickness in the cingulate gyrus of patients with schizophrenia-spectrum disorder were investigated and compared to those of healthy controls. Given the aforementioned evidence that memoryrelated activation occurs throughout the anterior-posterior axis of the cingulate gyrus and that duration of illness is associated with memory performance, we assessed cortical thickness abnormalities in different sub-areas of the cingulate cortex of patients with schizophrenia-spectrum disorder and its relation to memory as measured with different neuropsychological tests, and the duration of illness.

\section{Methods}

\section{Subjects}

In the present study, we report data from the POSITIVE study, a randomized-controlled multicenter clinical trial on the effectiveness of a cognitive behavioral treatment for persistent positive symptoms (delusion and hallucination) in psychosis [40]. All study participants were asked to participate in an additional protocol on magnetic resonance imaging (MRI) [42, 43]. Data from 67 patients with schizophrenia-spectrum disorder and 65 healthy control subjects matched for age, sex and education were analyzed. Inclusion criteria for patients were a DSM-IV diagnosis of schizophrenia (DSM-IV 295.1, 295.2, 295.3, 295.6, 295.9), schizophreniform disorder (DSM IV, 295.4), schizoaffective disorder (DSM-IV 295.7), or delusional disorder (DSM IV 297.1), confirmed by a structured clinical interview (SCID-I), PANNS-P1 Delusion or PANNS-P3 Hallucination score $\geq 4$ and persistent symptoms for at least 3 months. Patients and controls were recruited at six German university hospitals (Düsseldorf, Cologne, Bonn, Tübingen, Frankfurt and Essen). Controls had to have a life time history without psychiatric disorders, verified by a structured clinical interview (SCID-I, [81]. Overall, 41 subjects had to be excluded from the analysis because they had either incomplete data sets or produced errors in the FreeSurfer analysis pipeline or issues related to scan quality (details in Fig. 1). Quality assessment was done by visual inspection of the scans.

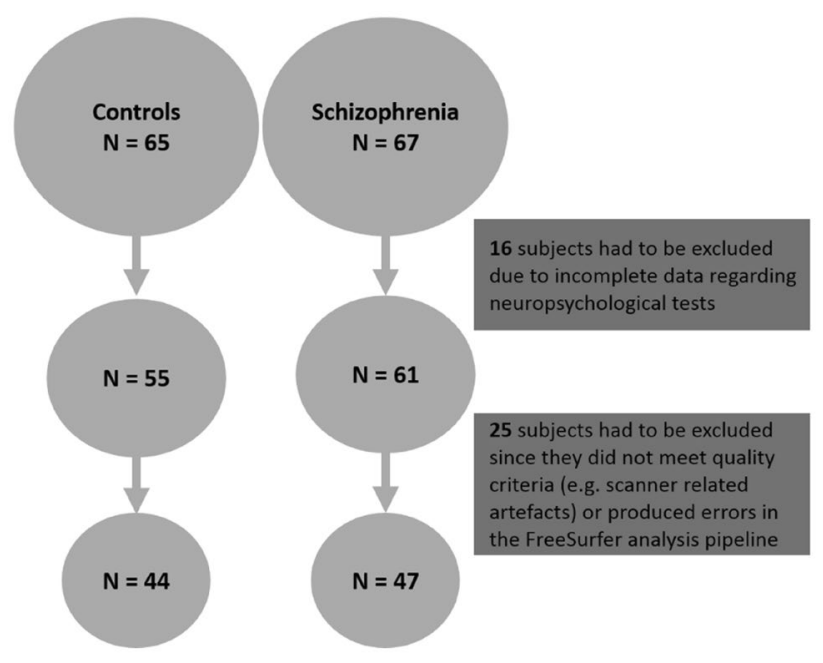

Fig. 1 Flow chart 
Subjects were excluded if there were issues such as no fullbrain coverage, artefacts as susceptibility, truncation, aliasing, chemical shift or ghosting and inadequate gray/white matter contrast.

A total of 47 patients with schizophrenia-spectrum disorder and 44 healthy controls were included and analyzed in the present study (see Table 1 for characteristics of the sample). All data reported here have been taken from the initial MRI assessment at the start of the psychotherapeutic intervention (see [40].

\section{Clinical data}

To account for potential differences regarding memory functions, we analyzed memory-related neuropsychological tests from the cognitive assessment battery in the POSITIVE study. These tests comprised the German Auditory Verbal Learning Test (VLMT; [33, 34, 48], the Digit Span Test [10], the Corsi Block-Tapping Test [16] and the Trail Making Test (TMT), Army Individual Test $[3,58]$. These tests allow the assessment of short-term memory (holding information in mind), working memory (holding information in mind and manipulating it and long-term memory functions. The VLMT is the German equivalent of the Rey Auditory Verbal Learning Test (RAVLT; [59] and designed to assess verbal episodic immediate and delayed memory, the latter with free recall and cued recognition. The Digit Span Test covers verbal short-term memory and working memory $[2,5,39,46,56$, 63] and consists of a forward memory part and a backward memory part for number sequences. The Digit Span forward is assumed to be more related to short term memory whereas the Digit Span backward is more related to working memory [15]. The Corsi Block-Tapping Test [29] probes visual-spatial short-term and working memory. Blocks on a rectangular board are tapped, which the subjects have to immediately replicate forward or backward. The Corsi Block-Tapping Test is assumed to inform about spatial short-term and working memory, respectively. The TMT consists of two parts,

Table 1 Sample characteristics

\begin{tabular}{lll}
\hline & $\begin{array}{l}\text { Schizophrenia } \\
\text { Mean (SD) }\end{array}$ & $\begin{array}{l}\text { Healthy controls } \\
\text { Mean (SD) }\end{array}$ \\
\hline Gender (male/female) & $37 / 10$ & $28 / 16$ \\
Age (years) & $37.49(8.20)$ & $35.57(9.37)$ \\
Education (MWT-B) & $27.17(4.73)$ & $31.33(3.53)$ \\
PANNS (positive scale) & $18.00(4.18)$ & $/$ \\
PANNS (negative scale) & $15.09(4.52)$ & $/$ \\
PANNS (general scale) & $32.74(6.84)$ & $/$ \\
Duration of illness (years) & $12.50(7.47)$ & $/$ \\
\hline
\end{tabular}

PANSS positive and negative syndrome scale, MWT-B MehrfachwahlWortschatz-Inteligenztest
TMT-A and TMT-B. In the TMT-A, subjects have to link numbers on a sheet of paper in ascending order. In part B numbers and letters have to be linked alternating in ascending order. TMT-A requires mainly visuoperceptual and motor speed abilities whereas the TMT-B reflects visuoperceptual, motor speed, working memory and executive functional abilities. Subtracting part A from B minimizes visuoperceptual and motor speed demands, providing a relatively better estimate of working memory abilities [47]. The MehrfachwahlWortschatz-Test is a word identification test (MWT-B; [45]. Real words with increasing difficulty have to be identified among five alternatives. The MWT-B is utilized to estimate subject's premorbid intelligence (verbal IQ).

To assess the relation of illness duration to potential alterations in cortical thickness and memory performance, we subtracted the subject's age at the patient reported start of psychotic symptoms from the subject's age at time of examination.

\section{MRI data acquisition}

Image acquisition was performed on $3 \mathrm{~T}$ MR scanners (all Siemens Healthcare, Germany) at 4 sites, i.e. Tübingen, Frankfurt, Jülich and Düsseldorf (all Germany), assessing patients recruited at 6 university centers (Tübingen, Heidelberg, Frankfurt, Bonn, Düsseldorf and Essen). A T1 weighted MPRAGE sequence was used for structural image acquisition (sagittal) with a $\mathrm{TR}=2.2 \mathrm{~s}, \mathrm{TE}=3.93 \mathrm{~ms}$ (minimal, asymmetric echo, bandwidth $130 \mathrm{~Hz} / \mathrm{Pixel}$ ), flip angle $=9^{\circ}$, slice thickness $=1.0 \mathrm{~mm}$, slices per slab $=160$, resolution $($ voxel size $)=1.0 \mathrm{~mm}^{3}$, FOV $256 \mathrm{~mm} \times 256 \mathrm{~mm}$.

\section{MRI data analyses}

FreeSurfer image analysis suite (version 6.0) was used for structural data processing. (http://surfer.nmr.mgh.harvard. edu/). Cortical thickness measures, generated by FreeSurfer, have been validated both by histological [62] and manual measurements [44]. Technical details have been described previously in Fischl and Dale [25]. Briefly, cortical thickness measures were obtained by reconstructing representations of the pial surface and the white/gray matter boundary [19,20] calculating the distance between those surfaces (Fig. 2B). Following automatic data processing, each image was manually inspected for errors in the processing pipeline.

\section{Description of regions of interest (ROI)}

The 2009 (V6) version of the FreeSurfer package implements a new parcellation of the cingulate cortex (Destrieux Atlas/ aparc.a2009s atlas; [21] based on cytoarchitectonic and functional data. The cingulate sulcus, cingulate gyrus and intracingulate sulcus were grouped and subdivided in 

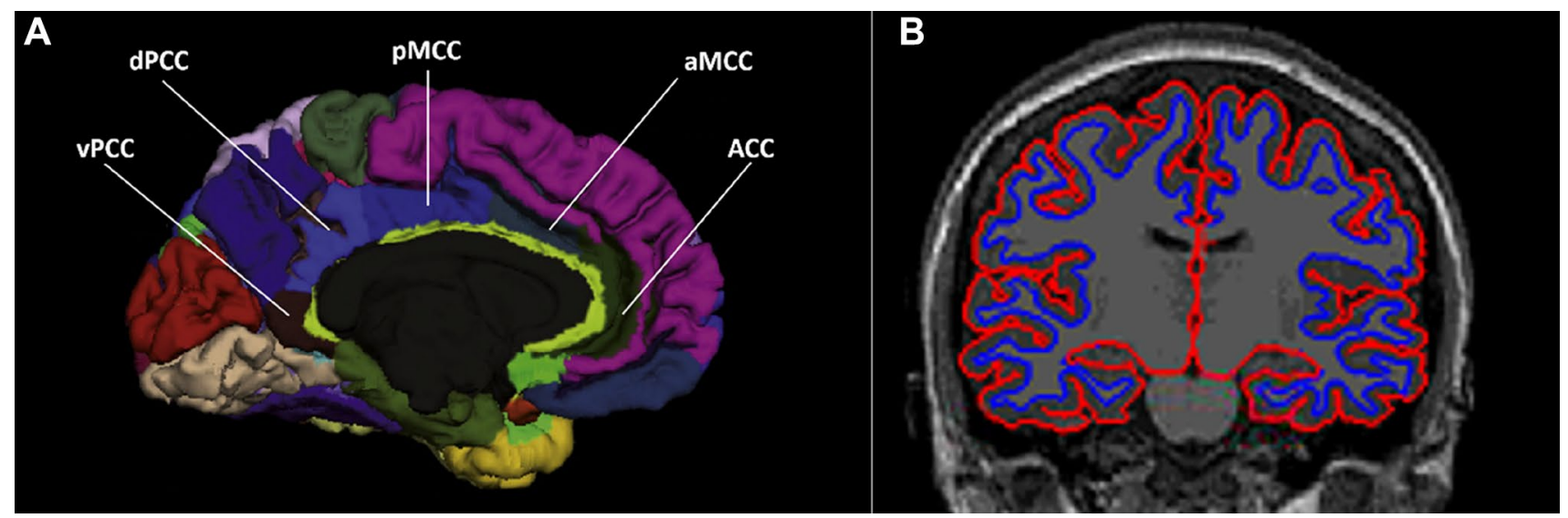

Fig. 2 (A) The cingulate ROI's are depicted. Anterior cingulate cortex (ACC; green), middle-anterior cingulate cortex (aMCC; gray), middle-posterior cingulate cortex (pMCC: dark blue), posterior-dorsal cingulate cortex (dPCC; light blue), posterior-ventral cingulate cortex (vPCC: brown). Figure adapted from [21]. (B) An image of

segments following the antero-posterior axis as proposed by Vogt and colleagues [77, 78]. This parcellation of the cingulate region includes an anterior (ACC), middle-anterior (aMCC), middle-posterior (pMCC), posterior-dorsal (dPCC), and posterior-ventral (vPCC or isthmus) subregion for each hemisphere (Fig. 2A).

\section{Statistical analysis}

The cingulate specific (aparc.a2009s atlas) output from the FreeSurfer analysis was subjected to SPSS (IBM Inc.) software for statistical analysis. Multivariate analysis of covariance (MANCOVA) was conducted to identify differences in cortical thickness between the patients with schizophrenia-spectrum disorder and healthy controls. Gender, age, study center and mean cortical thickness served as covariates of no interest. Cortical thickness measures of the left and right $\mathrm{ACC}$, aMCC, pMCC, dPCC, and $\mathrm{vPCC}$ served as independent and group as dependent variables. Associations of regional cortical thickness with duration of illness was assessed via Pearson's partial correlation analyses controlled for age and mean cortical thickness. In a next step, memory performance and its relation to cortical thickness was assessed. A MANCOVA was conducted to reveal memory differences between patients with schizophrenia-spectrum disorder and healthy control persons. Here, the neuropsychological tests, VLMT (immediate recall, delayed recall, delayed recognition), Digit Span (forward, backward), Corsi Block-Tapping (forward, backward) and TMT (A, B, B-A), served as independent and group as dependent variables. To determine effects related to gender, age, study center and education (as measured with the MWT-B), we used these as covariates of no interest. cortical thickness is depicted. The red lines represent the pial surface and the blue lines represent the gray-white matter border. Cortical thickness is defined as the distance between the red and blue lines orthogonal to the pial surface

Pearson's partial correlation between the neuropsychological tests and cortical thickness measures were calculated, controlling for age, mean cortical thickness and education. To reduce the amount of statistical tests, we assessed only associations between those cingulate subregions and neuropsychological tests that revealed a significant group difference in the initial MANCOVA analyses. In addition, we analyzed whether the neuropsychological tests that revealed a significant group difference were associated with illness duration. Therefore, we performed Pearson's partial correlation analysis controlled for age and education. We used Bonferroni correction for multiple testing.

\section{Results}

\section{MRI analysis}

The omnibus test (MANCOVA) regarding cortical thickness differences across the cingulate sub-regions revealed a significant main effect of group between patients and healthy controls $(F(10,76)=1.931, P=0.05)$. Post-hoc tests revealed significant differences between the independent groups: increased cortical thickness in the patients group in the right $\mathrm{vPCC}(F=5.208, P=0.025)$, and decreased cortical thickness in the patients group in the left ACC $(F=3.677, P=0.05)$ and the left aMCC $(F=13.359$, $P=0.001$ ) (Table 2). However, if corrected for multiple comparisons (Bonferroni correction), only the difference in the left aMCC remains significant. While, the inclusion of covariates as age, gender and mean cortical thickness can dramatically influence results [37] we reanalyzed the data without these covariates and found that the effects in the 
Table 2 Mean cortical thickness, in patients with schizophrenia-spectrum disorder and controls

\begin{tabular}{lllll}
\hline & $\begin{array}{l}\text { Schizophrenia } \\
\text { Estimated marginal } \\
\text { mean/SE (mm) }\end{array}$ & $\begin{array}{l}\text { Healthy controls } \\
\text { Estimated marginal } \\
\text { mean/SE (mm) }\end{array}$ & $F$ value & $P$ value \\
& $2.555 / 0.019$ & $2.548 / 0.020$ & 0.051 & 0.822 \\
Right anterior cingulate (ACC) & $2.621 / 0.019$ & $2.656 / 0.020$ & 1.142 & 0.237 \\
Right middle-anterior cingulate (aMCC) & $2.556 / 0.017$ & $2.598 / 0.018$ & 2.575 & 0.112 \\
Right middle-posterior cingulate (pMCC) & $2.910 / 0.021$ & $2.913 / 0.022$ & 0.006 & 0.939 \\
Right posterior-dorsal cingulate (dPCC) & $2.758 / 0.030$ & $2.639 / 0.030$ & 5.208 & $0.025^{*}$ \\
Right posterior-ventral cingulate (vPCC) & $2.708 / 0.021$ & $2.768 / 0.021$ & 3.677 & $0.050^{*}$ \\
Left anterior cingulate (ACC) & $2.584 / 0.024$ & $2.715 / 0.025$ & 13.358 & $0.001^{* *}$ \\
Left middle-anterior cingulate (aMCC) & $2.551 / 0.017$ & $2.585 / 0.018$ & 1.636 & 0.204 \\
Left middle-posterior cingulate (pMCC) & $2.983 / 0.023$ & $2.987 / 0.023$ & 2.054 & 0.155 \\
Left posterior-dorsal cingulate (dPCC) & $2.700 / 0.041$ & $2.660 / 0.042$ & 0.423 & 0.517 \\
Left posterior-ventral cingulate (vPCC) & & & \\
\hline
\end{tabular}

Means represent cortical thickness in millimeters $(\mathrm{mm})$. Compared to healthy controls, patients with schizophrenia-spectrum disorder showed reduced cortical thickness in the left anterior (ACC) and middle anterior cingulate $(\mathrm{aMCC})$ and increased cortical thickness in the right posterior ventral cingulate (vPCC)

*Significant difference

**Significant difference (Bonferroni corrected) left ACC remains stable (left ACC, $F=8.199, P=0.005$ / left aMCC; $F=20.428, P<0.001)$ but the effect in the right ACC turn to not significant (right vPCC; $F=3.231$, $P=0.07)$.

\section{Analysis of neuropsychological tests}

The omnibus test (MANCOVA) with neuropsychological tests as dependent variables revealed a significant main difference between patients and controls $(F(10,76)=4.884$, $P=0.001)$. Post hoc tests showed significant differences in the adjusted means between the independent groups for the Corsi Block-Tapping backward, VLMT- immediate recall, VLMT- delayed recall, VLMT- recognition, TMTA, TMT-B and TMT B-A. Compared to the healthy controls, patients performed worse on all these tests (Table 3). If corrected for multiple comparison (Bonferroni correction) all comparisons, except VLMT delayed recognition, remained significant.

\section{Correlation analysis}

Partial correlation analyses, revealed a positive association between duration of illness and cortical thickness measures in the right $\mathrm{vPCC}(r=0.300 ; P=0.050)$ but no associations with measures of left aMCC and ACC. The association between duration of illness and cortical thickness in the right $\mathrm{vPCC}$ remained even if additionally controlled for use of medication as measured with CPZ Equivalents. However, after correction for multiple testing (Bonferroni) no
Table 3 Estimated means of the neuropsychological tests, as corrected for gender, study center, age and education, of patients and healthy controls

\begin{tabular}{lllll}
\hline & $\begin{array}{l}\text { Schizophrenia } \\
\text { Estimated mar- } \\
\text { ginal mean/SE }\end{array}$ & $\begin{array}{l}\text { Healthy controls } \\
\text { Estimated mar- } \\
\text { ginal mean/SE }\end{array}$ & $F$ value & $P$ value \\
\hline Digit Span forward (amount correct) & $7.767 / 0.317$ & $7.784 / 0.330$ & 0.001 & 0.973 \\
Digit Span backward (amount correct) & $6.920 / 0.346$ & $7.714 / 0.360$ & 2.199 & 0.142 \\
Block-Tapping forward (amount correct) & $8.157 / 0.310$ & $8.343 / 0.322$ & 0.150 & 0.699 \\
Block-Tapping backward (amount correct) & $7.362 / 0.297$ & $9.009 / 0.309$ & 12.843 & $0.001^{* *}$ \\
VLMT immediate recall (amount remembered) & $9.465 / 0.437$ & $11.991 / 0.454$ & 13.989 & $0.001^{* *}$ \\
VLMT delayed recall (amount remembered) & $8.378 / 0.463$ & $12.084 / 0.481$ & 26.826 & $0.001^{* *}$ \\
VLMT delayed recognition (amount remembered) & $13.197 / 0.261$ & $14.347 / 0.271$ & 8.121 & $0.006^{*}$ \\
TMT-A (seconds) & $33.108 / 1.471$ & $26.303 / 1.529$ & 8.948 & $0.004^{* *}$ \\
TMT-B (seconds) & $94.958 / 6.142$ & $61.324 / 6.365$ & 12.514 & $0.001^{* *}$ \\
TMT B-A (seconds) & $61.850 / 5.582$ & $35.020 / 5.803$ & 9.660 & $0.003^{* *}$ \\
\hline
\end{tabular}

*Significant difference

**Significant difference (Bonferroni corrected) 
significant results remained. With regard to memory, partial correlation analyses in patients with schizophrenia-spectrum disorder revealed no correlation between duration of illness and the memory measures that appeared to be affected in patients. Regarding cortical thickness, we found a positive partial correlation between VLMT delayed recall performance and cortical thickness measures of the left aMCC $(r=0.232 ; P=0.041)$. However, after correction for multiple testing (Bonferroni) no significant results remained. While the TMT (B minus A) data deviated from normal distribution, we performed Kendall's tau correlation and found negative correlation between TMT (TMT-B minus TMT-A) and cortical thickness measures of the left aMCC $(r=-0.317$; $P=0.002$; Fig. 3), which survived correction for multiple testing (Bonferroni). Similar to Assunção Leme et al. [4], there was no association between cortical thickness/performance measures and age of onset of the illness.

\section{Discussion}

In the present study, we found that patients with schizophrenia-spectrum disorder exhibited both regional increase and decrease of the cingulate cortex in different sub-regions. In the posterior part of the cingulate gyrus, patients showed increased cortical thickness positively related to illness duration whereas the decreased cortical thickness in anterior parts of the cingulate gyrus appeared to be unrelated to illness duration. However, it should be noted that the finding of increased posterior cingulate cortical thickness did not survive the Bonferroni correction for multiple testing and should therefore be treated with caution. Nevertheless, this finding may point to a new hypothesis of an association between cortical thickness in the posterior cingulate and illness duration in patients with schizophrenia-spectrum disorder. Moreover, episodic and working memory deficits of patients with schizophrenia-spectrum disorder appeared to be associated with cortical thickness changes in the anterior but not posterior parts of the cingulate gyrus.

Consistent with previous studies, we found that patients with schizophrenia-spectrum disorder showed decreased

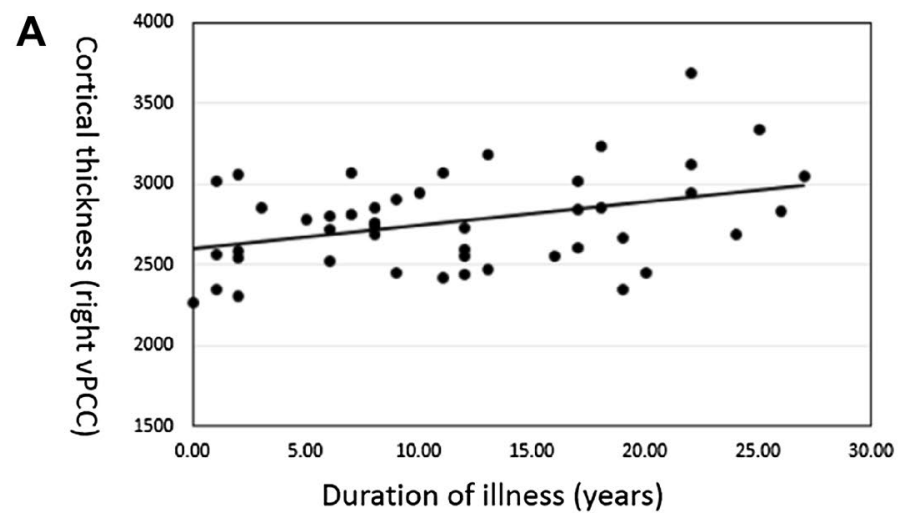

B

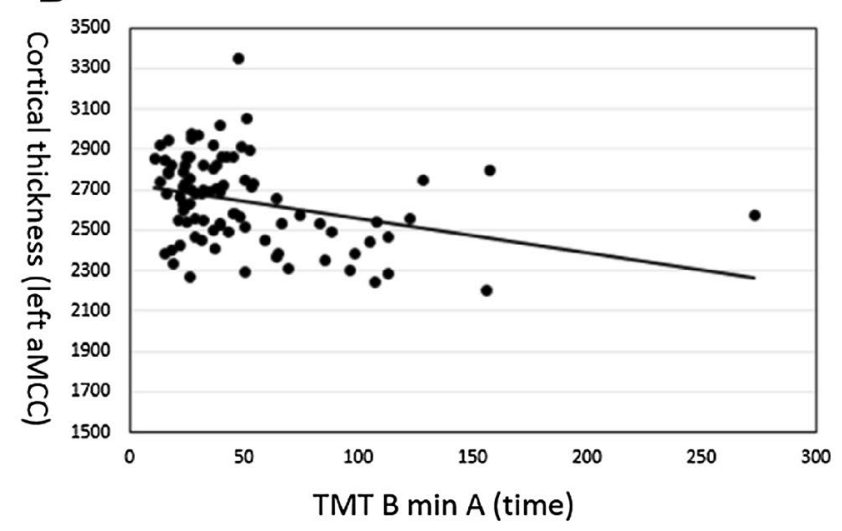

C

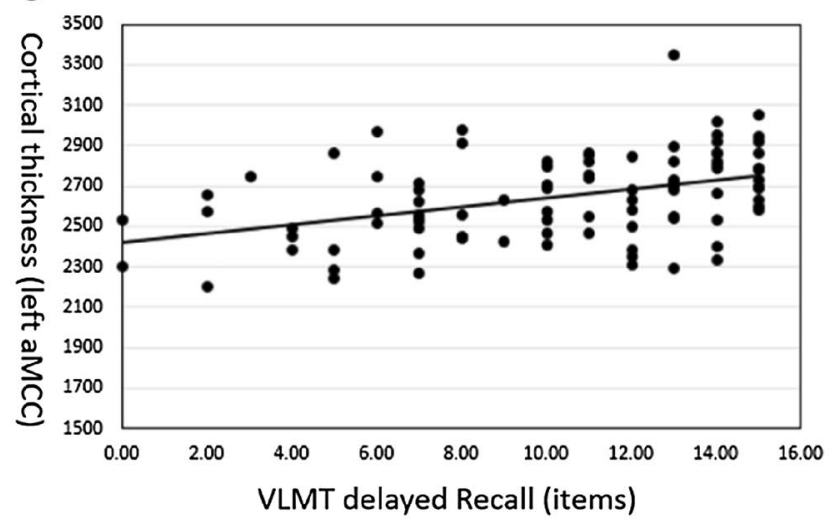

Fig. 3 Scatterplots of the significant correlations. In (A) the correlation between right vPCC cortical thickness and duration of illness, in (B) the correlation between left aMCC and TMT-B min (A) and in $(\mathbf{C})$ the correlation between left aMCC and VLMT delayed recall 
cortical thickness in anterior parts of the cingulate gyrus. This is in accordance with findings from Van Haaren et al. [75] who reported decreased anterior cingulate thickness in patients with schizophrenia-spectrum disorder that continued to decrease over the time course of five years. Interestingly, similar to our findings of increased cortical thickness in the right $\mathrm{vPCC}$, these authors showed that patients with schizophrenia-spectrum disorder exhibited increased cortical thickness in posterior parts of the brain such as parietal and occipital cortical areas. A hypothesis derived from these findings is that patients with schizophrenia-spectrum disorder may show a pronounced "communication" and neural processing within posterior brain networks, resulting in increased cortical thickness.

In line with this assumption is a recent finding by Wang et al. [80]. The authors studied resting-state-functional connectivity across several subregions of the cingulate gyrus in patients with schizophrenia-spectrum disorder. They reported that patients showed increased functional connectivity between posterior cingulate regions and parietal and occipital cortices, whereas anterior cingulate regions showed reduced functional connectivity to more frontal cortical regions. Functionally, Kronbichler et al. [41] reported decreased activation in anterior and increased activation in posterior brain regions of patients with schizophrenia during Theory of Mind tasks. In addition, empirical studies have demonstrated that activity of neurons can directly affect neurite outgrowth [76]. Taken together, the statistically uncorrected effect of increased cortical thickness in the vPCC may be taken to suggest that the "communication" of this region with others is increased, accompanied by augmented neuronal plasticity causing increased cortical thickness in posterior brain networks in which the vPCC functions as a hub. Another possible explanation may be related to maladaptive neurodevelopment in schizophrenia patients. The neurodevelopmental model of schizophrenia states that schizophrenia arises from insufficient synaptic pruning [24, 27, 57]. Insufficient synaptic or axonal pruning leaves more synapses or axon branches intact and could thereby cause cortical thickening in schizophrenia [82]. However, this seems unlikely given the positive correlation between vPCC cortical thickness and illness duration. Even though the exact pathological mechanisms of cortical thickening in schizophrenia needs further research, our finding of a statistically uncorrected vPCC thickening in patients with schizophrenia-spectrum disorder may provide a new hypothesis related to the pathology underlying schizophrenia.

Regarding memory, we evaluated memory deficits in patients with schizophrenia-spectrum disorder utilizing neuropsychological tests assessing working memory and different aspects of episodic memory such as immediate and delayed free recall and delayed recognition. Whereas working memory refers to the temporary storage of information and its manipulation and organization, recognition and recall refer to re-accessing of events or information from the past. Recognition refers to our ability to "recognize" an event or piece of information as being familiar, while recall designates the retrieval of related details of the memory. In line with previous studies (e.g. [52], we found that the patients with schizophrenia-spectrum disorder performed worse on neuropsychological tests of working memory (TMT-B minus TMT-A; CORSI block tapping backwards) and episodic memory (VLMT immediate and delayed recall). Regarding the association between cortical thickness and episodic memory performance we found a positive correlation between left aMCC cortical thickness and delayed recall of verbal material (VLMT-delayed recall). Note however, this effect should be interpreted with caution since it did not survive correction for multiple testing. Moreover, there was no association between cortical thickness and immediate recall (VLMT immediate recall). A possible explanation for the absence of an association between immediate recall performance and anterior cingulate thickness may be the remoteness of the memory. In this regard, functional studies in rodents and humans demonstrated that the anterior cingulate cortex is more involved in the retrieval of remote but not of recent memories [11, 70, 71]. However, with respect to cortical thickness, previous studies report inconsistent findings regarding an association between anterior cingulate cortical thickness and VLMT/RAVLT delayed recall performance. For instance, Chang et al. [14] reported no association between anterior cingulate cortical thickness and RAVLT delayed recall performance in patients with mild cognitive impairment whereas Gefen et al. [18] found a positive association in healthy elderly. These inconsistencies may at least partly be related to different parcelations of the cingulate cortex across these studies. Regarding vPCC, we could not find any associations between cortical thickness and episodic memory performance. A possible explanation may be that the neuropsychological tests used are not sensitive enough since functional MRI studies indicate posterior cingulate activation when participants performed episodic memory tasks $[55,60]$.

Regarding working memory, we found a negative correlation between TMT (TMT-B minus TMT-A) performance and left aMCC cortical thickness. This is similar to the finding by Gefen et al. [18], who reported a negative association between TMT-B raw sores and mean (left and right) anterior cingulate cortical thickness in the elderly. With respect to posterior cingulate cortical thickness we could not find an association with the different working memory tasks (TMT B minus A; Corsi block tapping backwards). However, functional imaging studies revealed evidence that both anterior and posterior cingulate regions are involved when participants perform the TMT task. For instance, Zakzanis et al. [82] showed increased activation in anterior 
and posterior parts of the cingulate gyrus in the TMT-B minus TMT-A condition in healthy young adults. Moreover, by using voxel-based morphology (VBM), Biundo and colleagues [9] revealed a negative association between TMT-B minus TMT-A performance and gray matter volume in both posterior and anterior cingulate gyrus. Moreover, MacPherson et al. [49] showed an association between cortical thickness in the isthmus of the cingulate cortex corresponding to $\mathrm{VPCC}$ and TMT-B performance in the elderly. However, MacPherson and colleagues included 411 healthy elderly and found a relation between TMT-B and a cluster located in the isthmus not the mean cortical thickness of whole isthmus as studied in the present study. Thus, sample size and/or the methodological analysis differences may explain these inconsistencies. In conclusion, we replicated that patients with schizophrenia-spectrum disorder exhibit memory deficits across different domains (delayed recall and working memory) and that these deficits are related to abnormal cortical thickness in the cingulate gyrus.

With respect to duration of illness, we could not find an association between duration and memory performance. Moreover, we found no association between duration of illness and anterior cingulate regions. However, as mentioned above, we found hints towards a probable association between duration of illness and right vPCC cortical thickness. This finding is not in line with the finding of Wang et al. [79]. Wang et al. reported a trend towards a negative association between duration of illness and both anterior and posterior cingulate cortical thickness. Moreover, Kuperberg et al. [44] reported a significant negative correlation between anterior cingulate cortical thickness and duration of illness whereas Nesvag and colleagues [54], Schultz et al. [64] and Assunção Leme et al. [4] could not find an association between cingulate thickness and illness duration. Thus, studies assessing the association between duration of illness and cingulate cortical thickness are controversial. This may be related to methodological differences in estimating cortical thickness, the parcellation of the cingulate sub-regions and different statistical procedures controlling for age, sex and education. Nevertheless, our finding of a (statistically uncorrected) positive relation between duration of illness and cortical thickness in the vPCC may indicate that there are specific neuronal processes that are changed during the time course of schizophrenia causing abnormal increased neuronal plasticity as opposed to neurodegeneration.

Some limitations should be acknowledged in this study. First, even though results were controlled for scanning site, this does not control for signal to noise ratio fluctuation between scanners over time. Therefore, noise due to changes in scanner profiles may have influenced the results $[42,43]$. Second, patients were chosen based on their current psychopathology (predominant positive symptoms) and their interest in participation in a study on cognitive therapy in schizophrenia. While patients had a notable duration of illness, the validity of the results of our study may be confined to patients with some years in treatment and therefore not present in first episode patients. Third, we had to exclude some participants due to methodological issues. Fourth, this work compares many different variables leading to the issue of the possibility of type 1 errors. To prevent these, Bonferroni correction was applied which reduced significance of the results dramatically. Therefore, the validity of results that did not survive Bonferroni correction should be treated with caution. A strength of our study is the sampling of data within the context of a large phase III equivalent randomized controlled trial, with clinical ratings by dedicated researchers and professional data handling procedures [40].

Altogether we replicated findings of reduced cortical thickness in anterior parts of cingulate gyrus in patients with schizophrenia-spectrum disorder that are associated with both episodic and working memory deficits in these patients. Moreover, to the best of our knowledge, we showed for the first time that patients with schizophrenia-spectrum disorder may exhibit increased cortical thickness in the right vPCC that seems to be associated with duration of illness. Future studies may utilize functional MRI to assess whether patients with schizophrenia have pronounced neural processing within posterior brain networks and if this is related to cortical thickness of the isthmus cingulate.

Funding Open Access funding enabled and organized by Projekt DEAL. This research work was supported by Bundesministerium für Bildung und Forschung, grant number 01GV0618.

\section{Declarations}

Conflict of interest The authors declare no conflict of interest.

Open Access This article is licensed under a Creative Commons Attribution 4.0 International License, which permits use, sharing, adaptation, distribution and reproduction in any medium or format, as long as you give appropriate credit to the original author(s) and the source, provide a link to the Creative Commons licence, and indicate if changes were made. The images or other third party material in this article are included in the article's Creative Commons licence, unless indicated otherwise in a credit line to the material. If material is not included in the article's Creative Commons licence and your intended use is not permitted by statutory regulation or exceeds the permitted use, you will need to obtain permission directly from the copyright holder. To view a copy of this licence, visit http://creativecommons.org/licenses/by/4.0/.

\section{References}

1. Aleman A, Hijman R, De Haan EHF, Kahn RS (1999) Memory impairment in schizophrenia: a meta-analysis. Am J Psychiatry 156:1358-1366 
2. Anstey KJ, Smith GA (1999) Interrelationships among biological markers of aging, health, activity, acculturation, and cognitive performance in late adulthood. Psychol Aging 14:605-618

3. Battery AIT (1944) Manual of Directions and Scoring. War Department, Adjutant General's Office, Washington, DC

4. Assunção Leme IB, Gadelha A, Sato JR, Ota VK, Mari JJ, Melaragno MI et al (2013) Is there an association between cortical thickness, age of onset, and duration of illness in schizophrenia? CNS Spectr 18:315-321

5. Banken JA (1985) Clinical utility of considering digits forward and digits backward as separate components of the Wechsler Adult Intelligence Scale-revised. J Clin Psychol 41:686-691

6. Beckmann M, Johansen-Berg H, Rushworth MF (2009) Connectivity-based parcellation of human cingulate cortex and its relation to functional specialization. J Neurosci 29:1175-1190

7. Benes FM, Vincent SL, Todtenkopf M (2001) The density of pyramidal and nonpyramidal neurons in anterior cingulate cortex of schizophrenic and bipolar subjects. Biol Psychiatry 50:395-406

8. Bilder RM, Goldman RS, Volavka J, Czobor P, Hoptman M, Sheitman B et al (2002) Neurocognitive effects of clozapine, olanzapine, risperidone, and haloperidol in patients with chronic schizophrenia or schizoaffective disorder. Am J Psychiatry 159:1018-1028

9. Biundo R, Formento-Dojot P, Facchini S, Vallelunga A, Ghezzo L, Foscolo L et al (2011) Brain volume changes in Parkinson's disease and their relationship with cognitive and behavioural abnormalities. J Neurol Sci 310:64-69

10. Blankenship AB (1938) Memory span: a review of the literature. Psychol Bull 35:2-3

11. Bontempi B, Laurent-Demir C, Destrade C, Jaffard R (1999) Time dependent reorganization of brain circuitry underlying long-term memory storage. Nature 400:671-675

12. Bowie CR, Harvey PD (2006) Cognitive deficits and functional outcome in schizophrenia. Neuropsychiatr Dis Treat 2:531-536

13. Chana G, Landau S, Beasley C, Everall IP, Cotter D (2003) Twodimensional assessment of cytoarchitecture in the anterior cingulate cortex in major depressive disorder, bipolar disorder, and schizophrenia: evidence for decreased neuronal somal size and increased neuronal density. Biol Psychiatry 53:1086-1098

14. Chang YL, Bondi MW, Fennema-Notestine C, McEvoy LK, Hagler DJ Jr, Jacobson MW et al (2010) Brain substrates of learning and retention in mild cognitive impairment diagnosis and progression to Alzheimer's disease. Neuropsychologia 48:1237-1247

15. Choi HJ, Lee DY, Seo EH, Jo MK, Sohn BK, Choe YM et al (2014) A normative study of the digit span in an educationally diverse elderly population. Psychiatry Investig 11:39-43

16. Corsi PM (1972) Human memory and the medial temporal region of the brain. McGill University, Montreal

17. Cuesta MJ, Peralta V, Zarzuela A (1998) Illness duration and neuropsychological impairments in schizophrenia. Schizophr Res 33:141-150

18. Gefen T, Peterson M, Papastefan ST, Martersteck A, Whitney K, Rademaker A et al (2015) Morphometric and histologic substrates of cingulate integrity in elders with exceptional memory capacity. J Neurosci 35:1781-1791

19. Dale AM, Fischl B, Sereno MI (1999) Cortical surface-based analysis. Segmentation and surface reconstruction. Neuroimage 9:179-194

20. Dale AM, Sereno MI (1993) Improved localization of cortical activity by combining EEG and MEG with MRI cortical surface reconstruction: a linear approach. J Cogn Neurosci 5:162-176

21. Destrieux C, Fischl B, Dale A, Halgren E (2010) Automatic parcellation of human cortical gyri and sulci using standard anatomical nomenclature. Neuroimage 53:1-15

22. Elsabagh S, Premkumar P, Anilkumar AP, Kumari V (2009) A longer duration of schizophrenic illness has sex-specific associations within the working memory neuronal network in schizophrenia. Behav Brain Res 201:41-47

23. Emilien G, Durlach C, Antoniadis E, Van der Linden M, Maloteaux JM (2004) Memory: neuropsychological, imaging and psychopharmacological perspectives. Psychology Press, Hove

24. Feinberg I (1982) Schizophrenia: caused by a fault in programmed synaptic elimination during adolescence? J Psychiatr Res 17:319-334

25. Fischl B, Dale AM (2000) Measuring the thickness of the human cerebral cortex from magnetic resonance images. Proc Natl Acad Sci 97:11050-11055

26. Forbes N, Carrick L, McIntosh A, Lawrie S (2009) Working memory in schizophrenia: a meta-analysis. Psychol Med 39:889-905

27. Goghari VM, Rehm K, Carter CS, MacDonald AW (2007) Regionally specific cortical thinning and gray matter abnormalities in the healthy relatives of schizophrenia patients. Cereb Cortex 17:415-424

28. Gold JM, Randolph C, Carpenter CJ, Goldberg TE, Weinberger DR (1992) Forms of memory failure in schizophrenia. J Abnorm Psychol 101:487-494

29. Corsi PM (1970) Human memory and the medial temporal region of the brain. Ph.D. thesis. McGill University

30. Gur RC, Gur RE (2013) Memory in health and in schizophrenia. Dialogues Clin Neurosci 15:399-410

31. Ha TH, Youn T, Ha KS, Rho KS, Lee JM, Kim IY et al (2004) Gray matter abnormalities in paranoid schizophrenia and their clinical correlations. Psychiatry Res 132:251-260

32. Häfner H, Riecher-Rössler A, Hambrecht M, Maurer K, Meissner S, Schmidtke A et al (1992) IRAOS: an instrument for the assessment of onset and early course of schizophrenia. Schizophr Res 6:209-223

33. Helmstaedter C, Durwen HF (1990) The Verbal Learning and Retention Test. A useful and differentiated tool in evaluating verbal memory performance. Schweiz Arch Neurol Psychiatr 141:21-30

34. Helmstaedter C, Lendt M, Lux S (2001) VLMT: Verbaler Lernund Merkfähigkeitstest. Beltz Test GmbH, Göttingen

35. Hulshoff Pol HE, Kahn RS (2008) What happens after the first episode? A review of progressive brain changes in chronically ill patients with schizophrenia. Schizophr Bull 34:354-366

36. Hulshoff Pol HE, Schnack HG, Mandl RC, van Haren NE, Koning H, Collins DL et al (2001) Focal gray matter density changes in schizophrenia. Arch Gen Psychiatry 58:1118-1125

37. Hyatt CS, Max M, Owens MM, Crowe ML, Carter NT, Lynam DR, Miller JD (2020) The quandary of covarying: a brief review and empirical examination of covariate use in structural neuroimaging studies on psychological variables. J Neuroimage. https://doi.org/10.1016/j.neuroimage.2019.116225

38. Job DE, Whalley HC, McConnell S, Glabus M, Johnstone EC, Lawrie SM (2002) Structural gray matter differences between first-episode schizophrenics and normal controls using voxelbased morphometry. Neuroimage 17:880-889

39. Johnstone B, Erdal K, Stadler MA (1995) The relationship between the Wechsler Memory Scale Revised (WMS-R) attention index and putative measures of attention. J Clin Psychol Med Settings 2:195-204

40. Klingberg S, Wittorf A, Meisner C, Wölwer W, Wiedemann G, Herrlich J et al (2010) Cognitive behavioural therapy versus supportive therapy for persistent positive symptoms in psychotic disorders: the positive study, a multicenter, prospective, singleblind, randomised controlled clinical trial. Trials 11:123

41. Kronbichler L, Tschernegg M, Martin AI, Schurz M, Kronbichler M (2017) Abnormal brain activation during theory of mind tasks in Schizophrenia: a meta-analysis. Schizophr Bull 43:1240-1250 
42. Krug A, Cabanis M, Pyka M, Pauly K, Kellermann T, Walter H et al (2014) Attenuated prefrontal activation during decisionmaking under uncertainty in schizophrenia: a multi-center fMRI study. Schizophr Res 152:176-183

43. Krug A, Cabanis M, Pyka M, Pauly K, Walter H, Landsberg M et al (2014) Investigation of decision-making under uncertainty in healthy subjects: a multi-centric fMRI study. Behav Brain Res 261:89-96

44. Kuperberg GR, Broome MR, McGuire PK, David AS, Eddy M, Ozawa F et al (2003) Regionally localized thinning of the cerebral cortex in schizophrenia. Arch Gen Psychiatry 60:878-888

45. Lehrl S (2005) Mehrfachwahl-Wortschatz-Intelligenztest MWT-B. Spitta Verlag, Balingen

46. Lezak MD (1987) Norms for growing older. Dev Neuropsychol $3: 1-12$

47. Lezak MD, Howieson DB, Bigler ED, Tranel D (2012) Neuropsychological assessment, 5th edn. Oxford University Press, Oxford

48. Lux S, Helmstaedter C, Elger CE (1999) Normierungsstudie zum Verbalen Lern- und Merkfähigkeitstest (VLMT). Diagnostica 45:205-211

49. MacPherson SE, Cox SR, Dickie DA, Karama S, Starr JM, Evans AC et al (2017) Processing speed and the relationship between trail making test $\mathrm{B}$ performance, cortical thinning and white matter microstructure in older adults. Cortex 95:92-103

50. McKenna PJ, Tamlyn D, Lund CE, Mortmer AM, Hammond S, Baddeley AD (1990) Preliminary communication: amnestic syndrome in schizophrenia. Psychol Med 20:967-972

51. Mitelman SA, Shihabuddin L, Brickman AM, Hazlett EA, Buchsbaum MS (2003) MRI assessment of gray and white matter distribution in Brodmann's areas of the cortex in patients with schizophrenia with good and poor outcomes. Am J Psychiatry 160:2154-2168

52. Müller BW, Sartory G, Bender S (2004) Neuropsychological deficits and concomitant clinical symptoms in Schizophrenia. Eur Psychol 9:96-106

53. Narr KL, Toga AW, Szeszko P, Thompson PM, Woods RP, Robinson D et al (2005) Cortical thinning in cingulate and occipital cortices in first episode schizophrenia. Biol Psychiatry 58:32-40

54. Nesvåg R, Lawyer G, Varnäs K, Fjell AM, Walhovd KB, Frigessi A et al (2008) Regional thinning of the cerebral cortex in schizophrenia: effects of diagnosis, age and antipsychotic medication. Schizophr Res 98:16-28

55. Northoff G, Richter A, Bermpohl F, Grimm S, Martin E, Vl M et al (2005) NMDA hypofunction in the posterior cingulate as a model for schizophrenia: an exploratory ketamine administration study in fMRI. Schizophr Res 72:235-248

56. Orsini A, Chiacchio L, Cinque M, Cocchiaro C, Schiappa O, Grossi D (1986) Effects of age, education and sex on two tests of immediate memory: a study of normal subjects from 20 to 99 years of age. Percept Mot Skills 63:727-732

57. Rapoport JL, Giedd JN, Gogtay N (2012) Neurodevelopmental model of schizophrenia: update 2012. Mol Psychiatry $17: 1228-1238$

58. Reitan RM (1958) Validity of the trail making test as an indicator of organic brain damage. Percept Mot Skills 8:271-276

59. Rey A (1964) L'Examen Clinique en Psychologie. Presse Universitaire de France, Paris

60. Ries ML, Schmitz TW, Kawahara TN, Torgerson BM, Trivedi MA, Johnson SC (2006) Task-dependent posterior cingulate activation in mild cognitive impairment. Neuroimage 29:485-492

61. Rund BR (1989) Distractibility and recall capability in schizophrenics: a 4 year longitudinal study of stability in cognitive performance. Schizophr Res 2:265-275

62. Rosas HD, Liu AK, Hersch S, Glessner M, Ferrante RJ, Salat DH et al (2002) Regional and progressive thinning of the cortical ribbon in Huntington's disease. Neurology 58:695-701
63. Ryan JJ, Lopez SJ, Paolo AM (1996) Digit span performance of persons 75-96 years of age: base rates and associations with selected demographic variables. Psychol Assess 8:324-327

64. Schultz CC, Koch K, Wagner G, Roebel M, Nenadic I, Schachtzabel $C$ et al (2010) Complex pattern of cortical thinning in schizophrenia: results from an automated surface based analysis of cortical thickness. Psychiatry Res 182:134-140

65. Seidman LJ, Lanca M, Kremen WS, Faraone SV, Tsuang MT (2003) Organizational and visual memory deficits in schizophrenia and bipolar psychoses using the Rey Osterrieth complex figure: effects of duration of illness. J Clin Exp Neuropsychol 25:949-964

66. Shapleske J, Rossell SL, Chitnis XA, Suckling J, Simmons A, Bullmore ET et al (2002) A computational morphometric MRI study of schizophrenia: effects of hallucinations. Cereb Cortex 12:1331-1341

67. Sigmundsson T, Suckling J, Maier M, Williams S, Bullmore E, Greenwood K et al (2001) Structural abnormalities in frontal, temporal, and limbic regions and interconnecting white matter tracts in schizophrenic patients with prominent negative symptoms. Am J Psychiatry 158:234-243

68. Sowell ER, Levitt J, Thompson PM, Holmes CJ, Blanton RE, Kornsand DS et al (2000) Brain abnormalities in early-onset schizophrenia spectrum disorder observed with statistical parametric mapping of structural magnetic resonance images. Am J Psychiatry 157:1475-1484

69. Sponheim SR, Jung RE, Seidman LJ, Mesholam-Gately RI, Manoach DS, O'Leary DS, Ho BC et al (2010) Cognitive deficits in recent-onset and chronic schizophrenia. J Psychiatr Res 44:421-428

70. Takashima A, Petersson KM, Rutters F, Tendolkar I, Jensen O, Zwarts MJ et al (2006) Declarative memory consolidation in humans: a prospective functional magnetic resonance imaging study. Proc Natl Acad Sci USA 103:756-761

71. Thielen JW, Takashima A, Rutters F, Tendolkar I, Fernandez G (2015) Transient relay function of midline thalamic nuclei during long-term memory consolidation in humans. Learn Mem 22:527-531

72. Todtenkopf MS, Vincent SL, Benes FM (2005) A cross-study meta-analysis and three-dimensional comparison of cell counting in the anterior cingulate cortex of schizophrenic and bipolar brain. Schizophr Res 73:79-89

73. Toulopoulou T, Morris RG, Rabe-Hesketh S, Murray RM (2003) Selectivity of verbal memory deficit in schizophrenic patients and their relatives. Am J Med Genet B Neuropsychiatr Genet 116B:1-7

74. Tripathi A, Kar SK, Shukla R (2018) Cognitive deficits in schizophrenia: understanding the biological correlates and remediation strategies. Clin Psychopharmacol Neurosci 16:7-17

75. Van Haaren NE, Schnack HG, Cahn W, van den Heuvel MP, Lepage C, Collins L et al (2011) Changes in cortical thickness during the course of illness in schizophrenia. Arch Gen Psychiatry 68:871-880

76. Van Ooyen A, Van Pelt J, Corner MA (1995) Implications of activity dependent neurite outgrowth for neuronal morphology and network development. J Theor Biol 172:63-82. https://doi.org/10. 1006/jtbi.1995.0005

77. Vogt BA, Berger GR, Derbyshire SW (2003) Structural and functional dichotomy of human midcingulate cortex. Eur J Neurosci 18:3134-3144

78. Vogt BA, Vogt L, Laureys S (2006) Cytology and functionally correlated circuits of human posterior cingulate areas. Neuroimage 29:452-466

79. Wang L, Hosakere M, Trein JC, Miller A, Ratnanather JT, Barch DM et al (2007) Abnormalities of cingulate gyrus neuroanatomy in schizophrenia. Schizophr Res 93:66-78 
80. Wang D, Zhou Y, Zhuo C, Qin W, Zhu J, Liu H et al (2015) Altered functional connectivity of the cingulate subregions in schizophrenia. Transl Psychiatry 5:e575. https://doi.org/10.1038/ tp.2015.69

81. Xiao Y, Lui S, Deng W, Yao L, Zhang W, Li S et al (2015) Altered cortical thickness related to clinical severity but not the untreated disease duration in schizophrenia. Schizophr Bull 41:201-210
82. Zakzanis KK, Mraz R, Graham SJ (2005) An fMRI study of the Trail Making Test. Neuropsychologia 43:1878-1886 